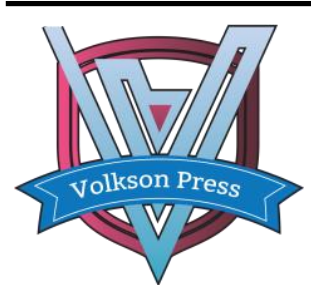

Contents List available at VOLKSON PRESS

Engineering \& Technology Innovations (ETI)

DOI : http://doi.org/10.26480/iceti.01.2017.123.125

\title{
ENGINEERING EXAMPLE OF DYNAMIC COMPACTION REPLACEMENT FOR MISCELLANEOUS FILL FOUNDATION AND ANALYSIS OF TREATMENT EFFECT
}

\author{
WU Daoxiang, HU Xueting, SHEN Qipeng, ZHANG Huijie \\ School of resources and environment engineering, HeFei University of Technology ,Hefei , China ,230009 \\ daoxiang_w@126.com
}

This is an open access article distributed under the Creative Commons Attribution License, which permits unrestricted use, distribution, and reproduction in any medium, provided the original work is properly cited

\section{ARTICLE DETAILS}

\section{ABSTRACT}

\section{Article History:}

Received 02 october 2017

Accepted 06 october 2017

Available online 11 october 2017

\section{Keywords:}

dynamic

bearing capacity
There is a layer of miscellaneous fill on the surface at a certain site in Huangshan City which has low strength, high compressibility and poor engineering properties. The dynamic replacement was used to increase its strength, reduce its compressibility, reduce the settlement and uneven settlement and meet the design requirements. In order to more greatly improve the bearing capacity of composite foundation and reduce the settlement of foundation, the dry hard concrete with low strength was used as replacement materials what was different from other dynamic replacement treatment projects where granular materials were used as replacement materials. The detailed treatment plan was made after trial ramming, and the dynamic replacement piers were tested through load tests and other tests after treatment. The test results showed that the dynamic replacement piers using dry hard concrete as replacement materials had high strength and low deformation which could improve the bearing capacity of composite foundation and reduce foundation settlement and uneven settlement greatly.

\section{INTRODUCTION}

Dynamic compaction method, also known as dynamic consolidation, was first proposed and applied by the French engineer L.Menard in 1969. Its main principle is to use a heavy hammer freely falling from a certain height and have a huge impact, which bear shock and vibration on foundation to make foundation structure impact, improving strength and reduce the deformation. It is a simple, low cost, significant effect, energy saving and environmental protection foundation treatment method.

In the early stage, the dynamic compaction method is mainly used for the treatment of miscellaneous fill, gravel soil, sandy soil and other foundations. Its is relatively simple for process to use lifting equipment to lift reciprocally a heavy hammer, free fall to tamp foundation and reinforce foundation. With the accumulation of experience and the progress of technology, the soil type scope for dynamic compaction method has made great extend. In addition to soil type which the early people think more suitable for dynamic compaction method, silt and muddy soil[1,2] and peat ${ }^{[3]}$ and other soft soil, expansive soi $\left.{ }^{[4]}\right]$ and loess soil[5,6] has even successful cases of dynamic compaction treatment. At the same time, the technology of the dynamic compaction method has made great progress and develop dynamic compaction replacement method[7], electroosmosis dynamic compaction method ${ }^{[8,9]}$, the explosion - dynamic compaction method ${ }^{[10]}$ and other new technology of dynamic compaction. In particular, it is proposed that tamping energy can have a much higher energy than those in the practical application to increase the reinforcement depth and improve treatment effect in recent years. The domestic biggest single-point tamping energy has broken through 15000 $\mathrm{kN} \cdot \mathrm{m}^{[11,12]}$.

\section{Engineering background}

The site is located in Tangkou Town, Huangshan District, Huangshan City, Anhui Province.There are extremely uneven, high compressibility of miscellaneous fill soil in the field. In order to accelerate the drainage consolidation of the base miscellaneous fill, improve the strength of the foundation soil and reduce its compressibility, improve the uniformity and density of the miscellaneous fill soil layer and reduce the settlement and settlement difference, the site was treated by dynamic compaction replacement method on the basis of the load characteristics of the superstructure and the site engineering geological condition.

Construction site is located in the middle and low mountain area, and the micro topography is floodplain topography on the right bank of the Fux river. Site fluctuation is large and the biggest difference is about $10 \mathrm{~m}$. The soil layers from top to bottom are (1)miscellaneous fill, (2)silt, (3)gravel, (4) boulder and (5)moderately weathered metamorphic sandstone:

(1)Miscellaneous fill: pale yellow, slightly wet, loose. The midwest of the site is mainly demolition and construction land, construction waste, domestic garbage and a small amount of powder soil and rubble backfill, The northeast region along the river is floodplain and mainly crushed stone and silty soil backfill. Its distribution of the soil layer is uneven and the thickness varies greatly between $0.40 \mathrm{~m}$ to $3.60 \mathrm{~m}$.

(2) Silt: yellow, slightly wet, loose. The soil layer and texture is uneven. The silt content is relatively high and the sand content at the bottom is higher. The thickness of the silt layer is uneven and between 0.2 to $2.10 \mathrm{~m}$. According to the test, the average blow count of SPT of the layer $\mathrm{N}=4$. Characteristic value of foundation bearing capacity $\mathrm{f}_{\mathrm{ak}}=110 \mathrm{kPa}$., and compression modulus Es=4.0MPa.

(3) Gravel: pale yellow, dry slightly wet, slightly dense dense. The main of its dense is micronesia. The particle shape is subangular angular. The skeleton particles are generally not in direct contact, which filled by coarse silt and medium coarse sand. Soil texture is uneven. Thickness is $0.2 \sim 1.90 \mathrm{~m}$. The heavy dynamic penetration value $\mathrm{N}_{63.5}=8.9 \sim 9.6$ and its average is 12.6 . Value of foundation bearing capacity $\mathrm{f}_{\mathrm{ak}}=160 \mathrm{kPa}$., and compression modulus Es=12.0MPa.

(4)Boulder: pale yellow, dry slightly wet, slightly dense $\sim$ dense. The main of its dense is micronesia. The particles shape is in a sub circle shape and the skeleton particles are generally not directly contacted, which are filled with sand and gravel. Soil texture is uneven. The heavy dynamic penetration value $\mathrm{N}_{63.5}=13.9 \sim 23.9$ and its average is 19.3. Value of foundation bearing capacity $\mathrm{f}_{\mathrm{ak}}=200 \mathrm{kPa}$, and compression modulus $\mathrm{Es}=15.0 \mathrm{MPa}$.

(5)Moderately weathered metamorphic sandstone: gray. The core was chunky. The rock is a fine grained clastic structure and medium thick layered structure, which is hard rock and is more complete. The basic quality grade of rock mass is III IVclass. The thickness of the layer exposed is $0.10 \sim 0.40 \mathrm{~m}$. The value of bearing capacity characteristic $\mathrm{f}_{\mathrm{ak}}=1000 \mathrm{kPa}$ and the compressibility is small. 


\section{Strengthening principle of dynamic consolidation replacement} method

Dynamic compaction replacement method is a foundation treatment method developed on the basis of dynamic compaction method. In the construction process of dynamic compaction, block stones, gravel, slag, construction wastes, and other hard coarse granular materials are filled into the tamper pits, and thus dense replacement piers are formed in the foundation through the hammer compaction. Replacement piers, soil between piers and sand-gravel cushion together form composite foundation, which improve the bearing capacity and reduce the settlement of the foundation.

Fig. 1 Schematic diagram of construction process of dynamic compaction

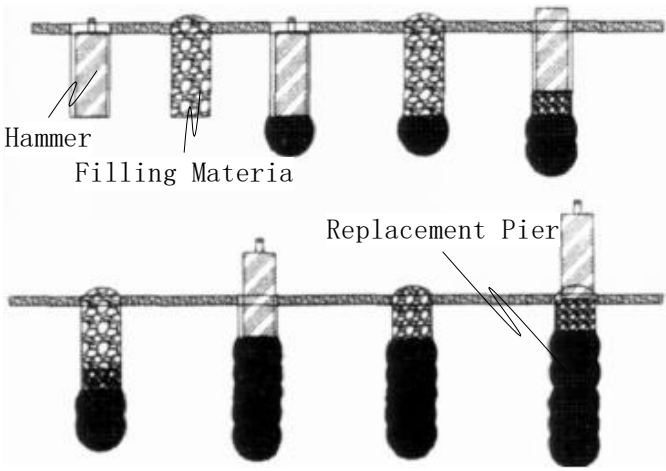

replacement method [13]

The action mechanism of dynamic consolidation replacement method is mainly:

(1)The replacement effect: compact replacement piers formed by dynamic compaction replace the original foundation soil.

(2)Compaction: The replacement piers laterally extrude those original foundation soil when the piers are forming, and the soil between replacement piers are enclosed.

(3) Consolidation: In the process of compaction, the impact energy provokes the foundation soil's drainage consolidation. The filling materials generally have good water permeability, which form good water permeable passages and is propitious for accelerating the consolidation of the foundation soil.

In the past, the replacement materials of dynamic replacement generally use granular materials. The increase range of the bearing capacity of the foundation is not high and the deformation is rare large generally after treatment. The miscellaneous fill in the site mainly contain residue soil producing while excavation of mountains in Tangkou Town, and some construction wastes because of house demolition. According to field conditions, dry hard concrete with low strength are used as replacement materials. Through high energy compaction, there is a large diameter of dry hard concrete replacement pier forming at the bottom of the column foundation.

\section{Dynamic Consolidation replacement program}

According to the preliminary tests, the dynamic consolidation replacement program is as follows:

The tamping points are arranged according to the column grid layout. The design pier length is $6.5 \mathrm{~m}$. The tamping energy is $2000 \mathrm{kN} \cdot \mathrm{m}$, the number of pounding is set to $20-28$ and the times of tamping is designed as one times of replacement tamping and two times of full compaction. The standards for end hammering is that The average compactive settlement of the last two ramming is less than $5.0 \mathrm{~cm}$, the settlement difference of the last two ramming is less than $3.0 \mathrm{~cm}$, and the uplift height is less than $100 \mathrm{~mm}$. The proportioning of the replacement material is cement: Sand: Gravel: water $=1: 2 \cdot 17: 4.17: 0.4$

\section{Detection and analysis of treatment effect}

28 days after the end of treatment, the load tests are adopted to test the capacity of single replacement pier, the load plate area is $2200 \mathrm{~mm} \times 2200 \mathrm{~mm}$, and the maximum test load is $6800 \mathrm{kN}$. The random sample of 3 replacement piers are extracted to test, and the test curves are as Fig.2 Fig.4
The curve showed that the inflection points of the load-settlement curves are obvious, and the curves of settlement load ratio corresponding to different levels of load also show the same pattern: When the load is less than the threshold pressure, the curve is relatively flat. But when the pressure exceeds the proportion limit, curve decline rapidly. It can be very clear to determine the proportion limit of each test, using this as the characteristic value of the bearing capacity of the single replacement pier $\mathrm{f}_{\mathrm{ak}}=4080 \mathrm{kN}$. The results show that the bearing capacity is rare high, the test data is close each other and the bearing capacity characteristic value determined by test are equal. This shows foundation treatment effect is rare good and the strength of the replacement pier is close after treatment, which is more favorable for the engineering.

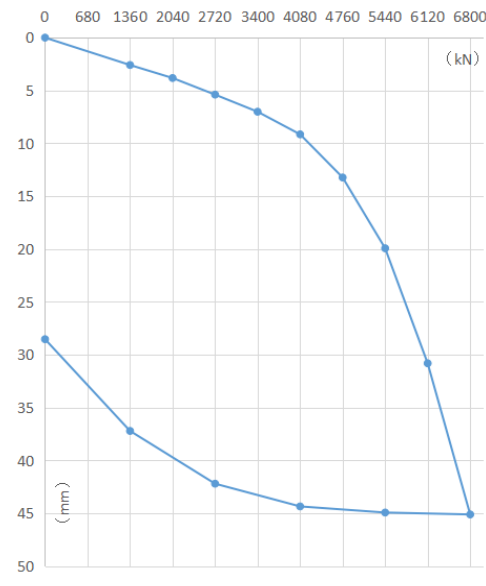

(a) load-settlement curve

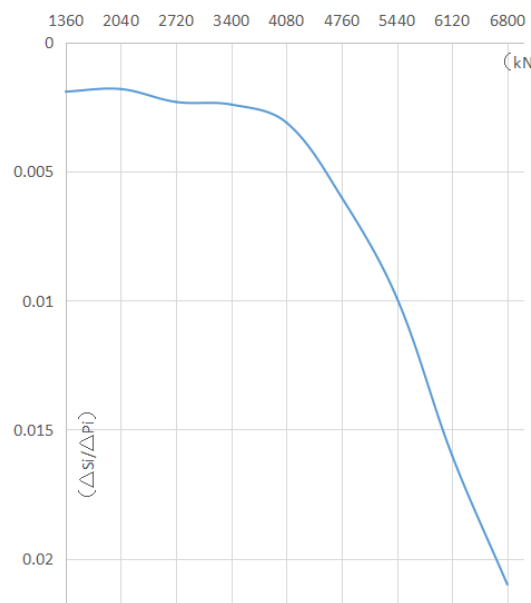

(b) settlement load ratio-load curve

Fig. 2 Test curves of test pier $1 \#$



(a) load-settlement curve 


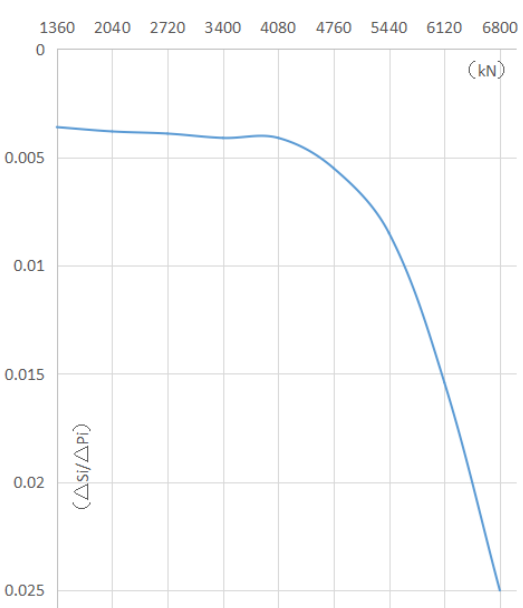

(b) settlement load ratio-load curve Fig. 3 Test curves of test pier 2\#

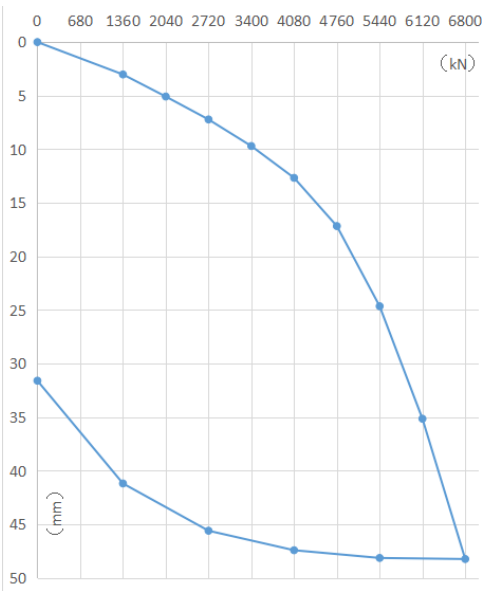

(a) load-settlement curve

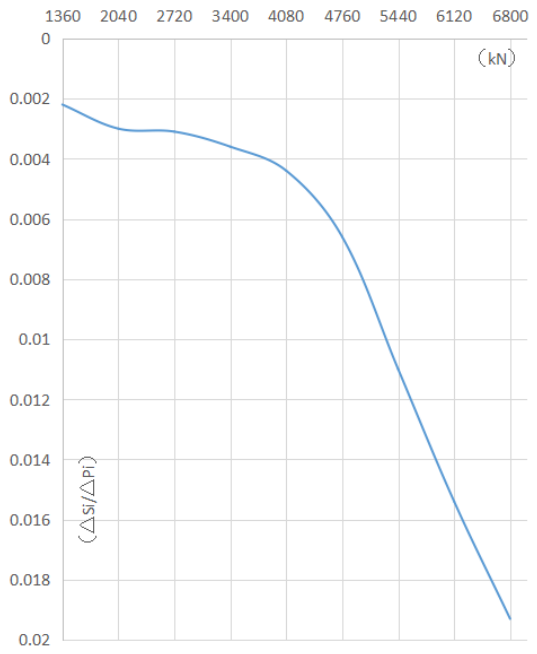

(b) settlement load ratio-load curve

Fig. 4 Test curves of test pier 3\#

\section{Conclusion}

According to the characteristics of the site, the dynamic compaction replacement foundation is chosen to deal with the miscellaneous fill site.
However, replacement material is used in granular materials in the past, and the bearing capacity after treatment is not high and the deformation is large generally. Therefore, the project adopted low strength dry hard concrete as replacement material. According to the results of load test after the treatment, the bearing capacity of single pile is high, up to $4080 \mathrm{kN}$, the deformation is small and the strength of pier after the treatment is close each other, which is conducive to reducing the differential settlement. Treatment and testing examples of this project show that dynamic replacement, whose replacement materials adopt low strength dry hard concrete, treating miscellaneous fill ground, have good effect, and the relevant results have a certain practical significance for the promotion and application of technology.

\section{Acknowledgements}

This work was funded by National Natural Science Foundation of China under Contract No. 41002117 and No. 40872190 .

\section{References}

[1] Wang Guoqiang, Li Yingxia, Wu Daoxiang, etc. "Effect analysis of heavy tamping treatment of foundation of a thick layer of recently deposited miscellaneous fill containing mud", Chinese Journal of Rock Mechanics and Engineering, 21(12), pp. 1868 1872(2002)

[2] Hu Xiuwen, Zhang Wei, Wang Jian. "Experimental study on dynamic compaction to improve saturated soft c1ay covered with hydraulic fill sand", Rock and Soil Mechanics, 25(5), pp. 818 823(2004)

[3] Zhang Huiping. "On treatment of peat clay foundation with dynamic semi-replacement", Shanxi Architecture, 37(32),pp. 57 58(2011)

[4] Shang Zhiyu, Li Weibin, Wu Weijie, etc. "Treatment of expansive soil foundation by dynamic compaction and dynamic compaction replacement", Cement Engineering, 28(6), pp. 83 85(2015)

[5] Zhang Changcheng, Ding Bing, Li Bin, etc. "Practice and analysis on collapsible loess treatment by using dynamic compaction with high energy", Geotechnical Investigation \& Surveying, (9), pp. 23 27(2013)

[6] Hu Changming, Mei Yuan, Wang Xueyan. "Experimental research on dynamic compaction parameters of collapsible loess foundation in Lishi region", Rock and Soil Mechanics, 33(10), pp. 2903 2909(2012)

[7] Zheng Lingwei, Zhou Fenghua. "Experimental study of forming process of replacement pier in soft soil using dynamic replacement method", Rock and Soil Mechanics, 35(1), pp. 91 98(2014)

[8] Zhao Jianguo, Zhu Wenkai. "A practice consolidating soft formations with an electric osmosizing-strong ramming composite method", Geology and Prospecting, 30(2),pp. 76 80(1994)

[9] Fu Hongtao, Wang Jun, Cai Yuanqiang, etc. "Experimental study of combined application of electro-osmosis and low-energy dynamic compaction in soft ground reinforcement", Chinese Journal of Rock Mechanics and Engineering, 34(3), pp. 612 620(2015)

[10] Yan Lin, Li Shihai, Zhang Jiahua. "A Project report of blasting-ramming combined densification of saturated sand", Soil Engineering and Foundation, 11(4), pp. 1 4(1997)

[11] Nian Tingkai, Li Hong jiang, Yang Qing, etc. "Improvement effect of high energy dynamic compaction under complicated geological conditions", Chinese Journal of Geotechnical Engineering, 31(1), pp. 141 146(2009)

[12] Nian Tingkai, Li Hong jiang, Yang Qing, etc. "Experiment of high energy dynamic compaction with $15000 \mathrm{kN} \cdot \mathrm{m}$ on a rubble fills site underlain by soft interlayer in coastal area", Rock and Soil Mechanics, 31(3), pp. $689 \sim 694(2010)$

[13] Zhao Yongchang, Hu Jianxun, Liu Yan, etc. "Analysis of Effect of Dynamic Compaction Replacement Treatment for Saturated Mucky Soil Foundation", Site Investigation Science and Technology, (6), pp. 36 39(2009) 